\title{
THE EFFECT OF PATIENT CONTROLLED LOCAL ANESTHETIC WOUND INFUSION ON POSTOPERATIVE PAIN IN CHILDREN UNDERGOING LAPAROTOMY
}

\section{Background and aims}

Local anesthetic wound infusions have shown promising results in reducing postoperative pain and the need of opioids in adults. But its use in children is still limited. Previously we found this method extremely effective for postoperative pain treatment in children undergoing orthopedic surgery. The aim of this report was to present the effectiveness of patient controlled local anesthetic wound infusion in children undergoing laparotomy.

\section{Methods}

Two patients (Table 1) in pediatric surgery department underwent laparotomy under general anesthesia and local anesthetic wound infusion was used for postoperative pain management. Catheter (IntraLong PAJUNK) was placed into the surgical wound. After the wound closure the PCA pump was connected and delivery of local anesthetic levobupivacaine $0.25 \%$ and $1.25 \mu \mathrm{g} / \mathrm{ml}$ of fentanyl solution was started. PCA devices settings are shown in figure below. Pain was evaluated using VAS scores every 3 hours, up to 48 hours when catheter was removed.

Table 1
\begin{tabular}{|l|l|l|}
\hline Cases & \multicolumn{1}{|c|}{ I I } \\
\hline Sex & Boy & Girl \\
\hline Age (years) & 10 & 10 \\
\hline Height (kg) & 35 & 29 \\
\hline ASA & III E & II \\
\hline Anesthesia & General & General \\
\hline Operation type & $\begin{array}{l}\text { Laparotomy, } \\
\text { adhesiolysis }\end{array}$ & $\begin{array}{l}\text { Laparotomy extirpation } \\
\text { ductus choledochus cyst, } \\
\text { hepatojejunostomy }\end{array}$ \\
\hline Operation time & 3h & 4h 55 min \\
\hline Anesthesia time & 3 h 20 min & 5 h 30 min \\
\hline
\end{tabular}

\section{Results}

Both patients received single dose of opioids before the end of anesthesia. Also NSAID and acetaminophen were given. Postoperatively both patients received additional treatment with NSAID once per day. VAS scores were $0-4$ (Table 2). No complications such as wound infection or systemic toxicity were reported.

Figure. Postoperative pain management

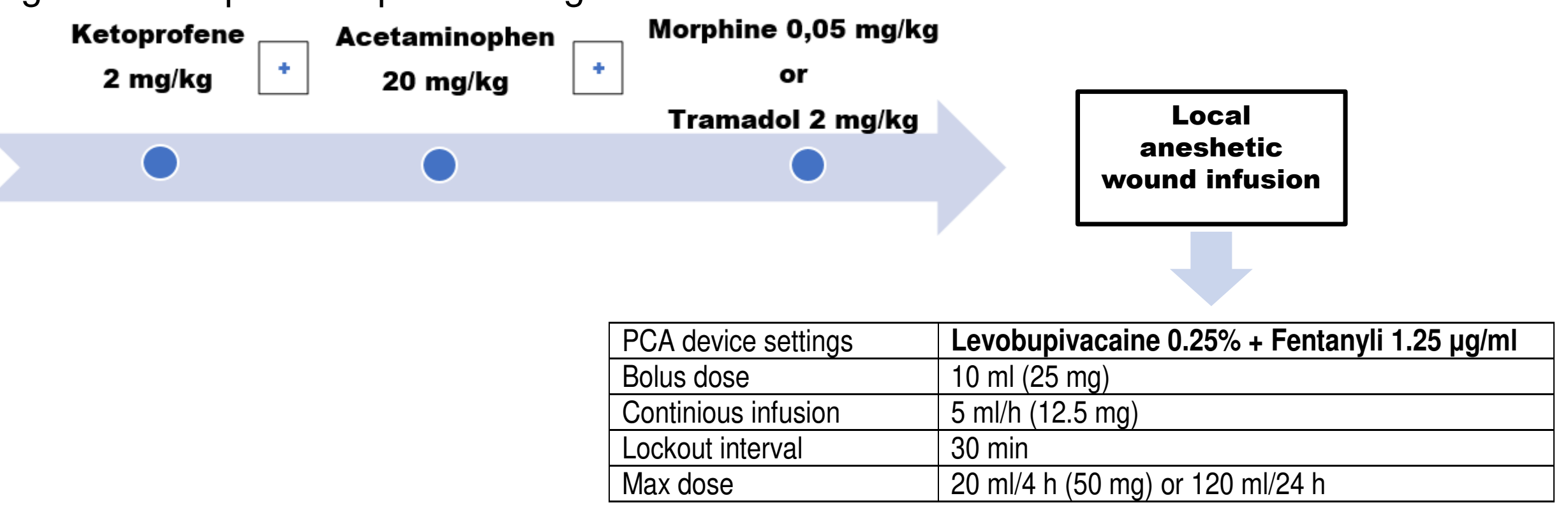

Table 2. Pain assessment
\begin{tabular}{|l|l|l|}
\hline \multirow{2}{*}{$\begin{array}{c}\text { Time, hours after } \\
\text { operation }\end{array}$} & \multicolumn{2}{|c|}{ Visual analogue scale } \\
\cline { 2 - 3 } & First case & Second case \\
\hline 0 & 0 & 0 \\
\hline 3 & 3 & 3 \\
\hline 6 & 0 & 1 \\
\hline 9 & 2 & 0 \\
\hline 12 & 0 & 4 \\
\hline 15 & 0 & 4 \\
\hline 18 & 0 & 4 \\
\hline 21 & 1 & 2 \\
\hline 24 & 4 & 4 \\
\hline 27 & 1 & $2-3$ \\
\hline 30 & 1 & $2-3$ \\
\hline 33 & 1 & 1 \\
\hline 36 & 1 & $3-4$ \\
\hline 39 & 0 & 1 \\
\hline 42 & 0 & 0 \\
\hline 45 & 0 & 0 \\
\hline 48 & 0 & 0 \\
\hline
\end{tabular}

\section{Discussion}

Continuous local anesthetic wound infusion is safe, simple technique, which can be used in many types of surgery, but there aren't enough studies with children (1). Some of these studies had limitations because of small sample size, different indications for surgery technique (2). Investigations have shown that surgical field infection and postoperative hernia incidence is very low.

\section{Conclusion}

Patient controlled wound infusion with local anesthetics was effective for postoperative pain treatment in pediatric patients undergoing laparotomy. The use of local anesthetics reduced total opioids requirements and improved pain scores. Less opioids requirement was associated with less opioid related side effects, better bowel recovery, shorter length of hospital stay, earlier mobilization. This method could be the best choice for postoperative pain treatment in children undergoing open abdominal surgery. 